\title{
Estrategias, tipos y grado de innovación: estudio en startups de tecnología de la información en el Noreste de Brasil
}

\section{Strategies, types and degree of innovation: study in information technology startups in Northeast Brazil}

\author{
Ronalty Oliveira Rocha Maestro en Administración (PROPADM/UFS). Universidade Federal de Sergipe (UFS) - Brasil. \\ ronaltyrocha@gmail.com \\ Maria Elena Leon Olave Doctor en Ingeniería de Producción. Universidade Federal de Sergipe (UFS) - Brasil. \\ mleonolave@gmail.com \\ Edward David Moreno Ordonez Doctor en Ingeniería Eléctrica. Universidade Federal de Sergipe (UFS) - Brasil. \\ edwdavid@gmail.com \\ Maria Conceição Melo Silva Luft Doctor en Administración. Universidade Federal de Sergipe (UFS) - Brasil. \\ ceicamelo.ufs@gmail.com \\ Luis Steven Calisaya Centty Estudiante universitário en Ciencias de la Educación. Universidad Nacional de San Agustín \\ (UNSA) - Peru. luis.calisayacentty@gmail.com
}

\section{RESUMEN}

Este artículo tuvo como objetivo presentar el grado de innovación, tipologías de estrategias para la innovación utilizadas por las startups de tecnología de la información en la región noreste de Brasil. A través de un estudio explicativo y exploratorio con un enfoque cuantitativo, esta investigación verifico especificamente cual es la tipología de estrategia de innovación más adoptada por las startups, así como analizo el grado de innovación en estas empresas, de acuerdo con el modelo llamado radar de innovación, usado por el SEBRAE. La recolección de las informaciones se llevo a cabo mediante el uso de una versión adaptada del formulário radar de innovación y el análisis de los datos consistió en una revisión de la estadística descriptiva y cálculo de las medias y desviación estándar de los datos recopilados. El análisis de los resultados indicó el predominio de las innovaciones de procesos como una práctica sistémica en startups de tecnología de la información. Con respecto a las estratégias para la innovación, se verifico la frecuencia de las asociaciones con clientes y con organizaciones como las principales practicas, mientras que la internacionalización fue la estrategia menos percibida. Con respecto al grado de innovación de startups de tecnologia de información, se encontró que las empresas consultadas estan classificadas como innovadoras medianas (ocasionales).

Palavras-chave: Startup. Estrategias de innovación. Asociaciones organizacionales. Asociaciones de clientes

\section{RESUMO}

Este trabalho teve como objetivo apresentar o grau de inovação em tipologias e estratégias para inovação utilizadas por startups de tecnologia da informação da Região Nordeste do Brasil. Por meio de um estudo explicativo e exploratório, de abordagem quantitativa, essa pesquisa pretendeu, especificamente, conferir qual a tipologia e estratégia de inovação mais adotadas por startups, assim como analisar o grau de inovação nessas empresas, segundo a metodologia do radar da inovação. A coleta de informações foi empreendida pelo uso de uma versão adaptada do formulário radar da inovação e a análise dos dados consistiu em avaliações de estatísticas descritivas e cálculo de médias e desvio-padrão dos dados coletados. A análise dos resultados indicou a predominância de inovações em processos como prática sistêmica nas startups de tecnologia da informação. No que se refere às estratégias para inovação constatou-se a frequência de parcerias com clientes e parcerias organizacionais como principais práticas, ao passo em que a internacionalização foi a estratégia menos percebida. Quanto ao grau de inovação das startups de tecnologia da informação verificou-se que as empresas consultadas estão classificadas como inovadoras medianas (ocasionais).

Palavras-chave: Inovação. Estratégias de inovação. Parcerias organizacionais. Parcerias com clientes.

\begin{abstract}
This article aimed to present the degree of innovation, typologies of strategies for innovation used by information technology startups in the northeast region of Brazil. Through an explanatory and exploratory study with a quantitative approach, this research specifically verified the type of innovation strategy most adopted by startups, as well as analyzing the degree of innovation in these companies, according to the model called radar of innovation, used by SEBRAE. The information collection was carried out through the use of an adapted version of the radar innovation form and the analysis of the data consisted of a review of the descriptive statistics and calculation of the means and standard deviation of the data collected. The analysis of the results indicated the predominance of process innovations as a systemic practice in information technology startups. With regard to innovation strategies, the frequency of partnerships with clients and organizations as the main practices was verified, while internationalization was the least perceived strategy. Regarding the degree of innovation of information technology startups, it was found that the companies consulted are classified as medium (occasional) innovators.
\end{abstract}

Keywords: Startup. Innovation strategies. Organizational partnerships. Customer partnerships. 


\section{INTRODUCCIÓN}

Porter (1996) ya indicó que la innovación es la principal estrategia competitiva para el reconocimiento de las oportunidades comerciales, supervivencia corporativa, formación de ventajas competitivas y para el progreso de la empresa, es decir, durante años, la innovación ha sido señalada como una de las formas más importantes para el mantenimiento y competitividad de las organizaciones.

En términos más generales, la innovación es la conversión de una idea nueva en algo que puede ser rentable para el progreso de las empresas, regiones y economías (Mas-Tur, Pinazo, Tur-Porcar, \& Sánchez-Masferrer, 2015). Según su definición habitual como herramienta para la inserción de nuevos conocimientos, la innovación brinda a las organizaciones que la adoptan el beneficio de atraer y mantener clientes al ofrecer productos nuevos y mejorados (Rocha, Olave, \& Ordonez, 2018).

Además, organizaciones practicantes de la innovación también pueden optimizar los procesos internos, reducir los costos de fabricación y el comercio de bienes, así como acceder a nuevos mercados (Camisón, \& Monfort -Mir, 2012). Por lo tanto, no es difícil asumir cuán relevante, independientemente del tamaño, sector y ubicación, es la práctica de la innovación en las organizaciones modernas.

En este escenario, Meireles, Picanço y Periotto (2018) destacan a las startups como negocios en evidencia para la inserción de innovaciones en el mercado. Esta percepción es corroborada por López, Del Rio y Lorente (2016) quienes refuerzan que las startups han sido destacadas por la inserción de productos y servicios nuevos o significativamente mejorados. Además, Galloway, Miller, Sahaym y Arthurs (2017) sostienen que las startups son un factor clave para la promoción de las innovaciones y el desarrollo de ideas, nuevos productos y servicios de alto valor agregado para las industrias existentes.

Sobre este tema, Andrade, Lins Filho e Silva (2016) afirman que las startups están en su mayoría compuestas por jóvenes emprendedores dispuestos a correr riesgos con ideas disruptivas, creativas e inovadoras, que crean nuevos negocios capaces de revitalizar la economía de un país. Por esta razón, Padrão y Andreassi (2013) corroboran la percepción de que las startups tienen un alto impacto en el desarrollo económico de una nación, ya que favorecen expresamente la generación de nuevos empleos.

La región noreste de Brasil, por su vez, se destaca cuando se trata de startups. Según los datos proporcionados por ABSTARTUPS (2018) y Accenture consultoría (2018), éste región alberga 557 empresas de esta naturaleza, lo que representa el $11 \%$ de startups de todo Brasil. De estos negocios, 119 empresas se clasifican como startups de tecnología de la información.

En ese contexto, dado el énfasis de la innovación como herramienta para lograr y mantener ventajas competitivas, ya sea en grandes, micro y pequeñas empresas o en startups, también es relevante estudiar cómo se idealiza la innovación en estas organizaciones, es decir, cuáles son las prácticas, procesos, rutinas, métodos de gestión y recursos invertidos para identificar oportunidades y comercializar novedades. Por lo tanto, se debe prestar atención a las estrategias de innovación. Las estrategias de innovación incluyen elegir los mejores y más apropiados planes organizacionales para el desarrollo e introducción de nuevos productos y servicios (Heidenreich, \& Kraemer, 2016).

Todavía en esta línea, la definición de una estrategia para la innovación debe ir acompañada de un análisis más detallado de los procesos de organización y características de funcionamiento, ya que la selección inadecuada de estrategias puede imposibilitar la práctica de la innovación en los negocios, incluyendo las startups, transformándolas en empresas con bajo impacto innovador, o convirtiéndolas incluso en negocios tradicionales que no producen innovaciones y solo comercializan productos estables.

Es importante resaltar que, al adoptar una estrategia para la innovación, las organizaciones también deben buscar medios y herramientas para probar y medir la efectividad de la estrategia adoptada frente a su desempeño comercial. En este contexto, las organizaciones necesitan de medios para medir su producción y capacidad innovadora, es decir, para medir su grado de innovación (Edison, Ali, \& Torkar, 2013). El grado de innovación refleja en cuánto la capacidad de innovación de una empresa se ha convertido en prácticas, productos y servicios innovadores (Jardón, 2012).

Dado lo anterior y considerando el potencial para el desarrollo económico y la creación de empleo en las startups, este artículo tiene como objetivo presentar el grado de innovación en tipologías y estrategias de innovación utilizadas por las startups de tecnología de la información en el noreste de Brasil. Específicamente, se buscó identificar, a través de la metodología de radar de innovación, los puntajes de práctica para tipologías y estrategias de innovación, así como medir el grado general de innovación en estos negocios.

Al exponer la relevancia de los temas propuestos, también está claro que, en Brasil, se publicaron pocos estudios sobre el tema de las estrategias de innovación asociadas con el grado de innovación en las startups, especialmente en la región noreste (según una encuesta realizada en la base de datos CAPES). 


\section{STARTUPS}

Gran parte de la literatura actual está usando la palabra startup de manera amplia y extendiéndolo a diferentes tipos de emprendimientos. Dávila y Foster (2005) caracterizan a las startups como compañías con menos de diez años de operación. Gelderen, Thurik y Bosma (2006), a su vez, consideran que las startups son todas compañías recién lanzadas, mientras que Nanda y Rhodes- Kropf (2013) creen que las startups son empresas financiadas con capital de riesgo.

El concepto de startups también puede entenderse como la tipificación de compañías temporales que buscan modelos comerciales repetibles, escalables y rentables (Blank, \& Dorf, 2012), diseñados para crear un nuevo producto/servicio, que operan a bajos costos iniciales, y condiciones de extrema incertidumbre (Hsieh, Lee, \& Huang, 2016).

A fin de resolver conflictos sobre el concepto de startups, el Cuardo 1 muestra siete elementos definitorios (tiempo de actividad; fuentes de financiación; tamaño y bajo costo de mantenimiento; tiempo de actividad y potencial de crecimiento; bajo costo de mantenimiento y potencial de crecimiento; base tecnológica y potencial de crecimiento; innovación y uso de herramientas tecnología) y sus respectivos conceptos.

\section{Cuadro 1.}

Conceptos de startups

\begin{tabular}{|c|c|c|}
\hline Elemento definitorio & Autor & Concepto \\
\hline \multirow{3}{*}{$\begin{array}{l}\text { Tiempo de } \\
\text { funcionamiento }\end{array}$} & Dávila y Foster (2005) & Empresas con menos de diez años. \\
\hline & Gelderen, Thurik y Bosma (2006) & Empresas recién lanzadas. \\
\hline & Alberti y Pizzurno (2017) & $\begin{array}{l}\text { Pequeña empresa que aún no está } \\
\text { establecida en el mercado y puede } \\
\text { fracasar fácilmente. }\end{array}$ \\
\hline $\begin{array}{l}\text { Fuentes de } \\
\text { Financiamiento }\end{array}$ & Nanda y Rhodes-Kropf (2013) & $\begin{array}{l}\text { Empresas financiadas con capital } \\
\text { riesgo. }\end{array}$ \\
\hline $\begin{array}{l}\text { Tamaño y bajo costo de } \\
\text { mantenimiento }\end{array}$ & Cicconi (2014) & $\begin{array}{l}\text { Pequeñas empresas de creación con } \\
\text { actividades de investigación y } \\
\text { desarrollo (P\&D), bajos costos de } \\
\text { mantenimiento y posibilidad rápida y } \\
\text { constante de crecimiento y } \\
\text { generación de ganancias. }\end{array}$ \\
\hline $\begin{array}{l}\text { Tiempo de ejecución y } \\
\text { potencial de crecimiento }\end{array}$ & Blank y Dorf (2012) & $\begin{array}{l}\text { Empresas temporales con modelos } \\
\text { de negocio repetibles y escalables. }\end{array}$ \\
\hline $\begin{array}{l}\text { Bajo costo de } \\
\text { mantenimiento y } \\
\text { potencial de crecimiento. }\end{array}$ & Hsieh, Lee y Huang (2016) & $\begin{array}{l}\text { Empresas creadas para lanzar nuevos } \\
\text { productos/servicios, que operan a } \\
\text { bajo costo y en condiciones de } \\
\text { incertidumbre. }\end{array}$ \\
\hline \multirow[t]{2}{*}{$\begin{array}{l}\text { Base tecnológica y } \\
\text { potencial de crecimiento. }\end{array}$} & Robehmed (2013); Shontell (2014) & $\begin{array}{l}\text { Empresas de base tecnológica con } \\
\text { alto potencial de crecimiento. }\end{array}$ \\
\hline & Miranda, Santos Junior y Dias (2016). & $\begin{array}{l}\text { Empresas que confían en la } \\
\text { innovación y el desarrollo } \\
\text { tecnológico para identificar } \\
\text { oportunidades de mercado con } \\
\text { productos/servicios innovadores y de } \\
\text { alto valor agregado. }\end{array}$ \\
\hline \multirow[t]{2}{*}{$\begin{array}{l}\text { Innovación y uso de } \\
\text { herramientas de } \\
\text { tecnología de la } \\
\text { información. }\end{array}$} & Ries (2012) & $\begin{array}{l}\text { Empresas que tienen la innovación } \\
\text { en su núcleo y quieren revolucionar } \\
\text { el mercado interactuando con los } \\
\text { clientes. }\end{array}$ \\
\hline & Stoilov (2015) & $\begin{array}{l}\text { Empresas diseñadas para crecer } \\
\text { rápidamente ayudadas por el uso de } \\
\text { herramientas de tecnología de la } \\
\text { información. }\end{array}$ \\
\hline
\end{tabular}

Fuente: Elaboración propia (2019). 
Dadas las diferentes definiciones de startups y de acuerdo con el análisis de la Tabla 1, este trabajo entendió que el término startup está vinculado a empresas de base tecnológica, recientemente lanzadas o que operan a corto plazo y que adoptan la innovación tecnológica como fuente de estrategia competitiva que opera en condiciones de incertidumbre, y que desarrollan modelos escalables y repetibles con potencial para un alto crecimiento empresarial a partir del desarrollo y lanzamiento de nuevos productos y servicios.

Dada la relevancia de estas empresas para promover la innovación, el siguiente tema presentará las principales prácticas estratégicas para la innovación adoptadas por las startups.

\subsection{Prácticas estratégicas para la innovación en startups}

Al definir una estratégia, también llamada práctica estratégica para la innovación, las organizaciones definen el papel de la innovación en su dirección estratégica (Cruz, Camargo, Brás, Frei y Frei, 2015). En resumen, las estrategias de innovación están representadas en la armoniosa reconciliación entre los objetivos de innovación y los objetivos estratégicos y organizativos (Crossan, \& Apaydin, 2010).

Además, Berne (2016) afirma que en las startups el proceso y la práctica de la innovación implica el intercambio de información, recursos y procedimientos técnicos entre diferentes agentes comerciales, ya sean internos o externos a la empresa, como clientes, proveedores e instituciones de ciencia y tecnología (Freire, Maruyama, \& Polli, 2017).

Desde este entendimiento, Rocha, Olave y Ordóñez(2018) descubrieron que las prácticas estratégicas de las asociaciones organizacionales, las asociaciones con los clientes, la innovación abierta y la internacionalización, son enfoques estratégicos más pertinentes para las startups.

Según la concepción de estos autores, se explica que las asociaciones organizacionales son prácticas estratégicas emprendidas entre organizaciones similares y complementarias que se asocian, a través de mecanismos formales $y / o$ informales, para acceder al conocimiento externo con el fin de obtener experiencia y ampliar la cartera de soluciones internas (Saebi, \& Foss, 2014; Alberti, \& Pizzurno, 2017).

Además de las asociaciones organizacionales, se argumenta que la práctica estratégica de asociarse con los clientes, que también se llama co-creación, se caracteriza por los esfuerzos cooperativos entre organizaciones y clientes para crear y mejorar nuevos bienes, ya sean productos o servicios (Cropley, Kaufman, \& Cropley, 2011).

Otra estrategia discutida en la literatura académica como promotora de la innovación es la innovación abierta (Agrawal, Bhattacharya, \& Hasija, 2016). Chesbrough (2012) atestigua que la innovación abierta se practica buscando y reteniendo conocimiento externo a través de la colaboración con clientes, proveedores, competidores y agentes institucionales para fusionar competencias internas con tecnologías externas con el fin de impulsar el potencial para introducir nuevos productos, servicios y procesos administrativos y operativos (Keinz, Hienerth, \& Lettl, 2012).

Por último, Shaw y Williams (2009) tienen a la internacionalización como práctica estratégica para la innovación, esto, porque las empresas exportadoras absorben el conocimiento técnico y estratégico de la interacción con contactos en el extranjero, fomentando de esta manera, la adopción de nuevas tecnologías de producción y, en consecuencia, mayor productividad y rendimiento empresarial (Ren, Eisingerich, \& Tsai, 2015).

Love y Roper (2015), que corroboran las prácticas estratégicas para la innovación, señalan tres canales principales a través de los cuales las pequeñas empresas pueden obtener conocimiento externo (asociaciones) que pueden contribuir a su actividad de innovación. El primer canal que los autores llaman Being There, ocurre cuando las empresas se benefician de los flujos de conocimiento local o de la información mediada por contactos sociales o enlaces al mercado laboral.

El segundo canal sugerido por los autores se llama Openess, en virtud de esta estrategia, las empresas entablan relaciones deliberadas, constituidas formalmente por vínculos de colaboración y contractuales (Glückler, 2013) con otras organizaciones, para reunir conocimiento técnico y comprensión del mercado. Finalmente, el tercer canal para obtener conocimiento externo se llama aprendizaje mediante la exportación, cuando las empresas aprenden nuevas prácticas de manera efectiva a través del contacto con mercados intensivos en conocimiento o altamente competitivos (Ganotakis, \& Love, 2011).

Como se puede ver, en las startups, las estrategias de innovación involucran la participación de diferentes actores y agentes internos y externos, revelando que el enfoque colaborativo, así como la interacción con diferentes audiencias, es uno de los principales instrumentos del proceso innovador en este tipo de negocios. 


\section{PROCEDIMIENTOS METODOLÓGICOS}

En cuanto a sus objetivos, este estudio fue considerado como una investigación exploratoria porque tiene como objetivo conocer y medir, en las startups, el grado de innovación en tipologías y prácticas estratégicas para la innovación, un tema poco abordado en la literatura brasileña para este tipo de negocios. El aspecto exploratorio de esta investigación se confirma mediante una encuesta realizada en la base de datos de las revistas CAPES (verificada en octubre de 2019), en la que no se identificaron estudios que aborden específicamente el grado de innovación en las startups.

Este estudio también es, aunque en menor medida, un estudio explicativo mediante la adopción de técnicas cuantitativas para el análisis de datos: resultados porcentuales, variabilidad de los datos obtenidos (desviación estándar - DE), grado y puntajes para tipologías y estrategias de innovación - de donde se solicitó aclaración sobre los factores que influyen en la práctica de la innovación en startups de tecnología de la información.

En cuanto a la forma de enfoque, esta investigación se presentó como cuantitativa, y se implementó mediante el uso de formularios electrónicos. El formulario en cuestión es una adaptación del formulario radar de innovación, utilizado y puesto a disposición por Berne (2016). El radar de innovación es una herramienta para medir el grado de innovación, desarrollado por Sawhney, Wolcott y Arroniz (2006) y luego adaptado a la realidad de las micro y pequeñas (MPEs) por Bachmann y Destafani (2008). Este instrumento se envió a los participantes de esta investigación en forma de cuestionario electrónico, dispuesto en versión digital con la ayuda de la herramienta Formularios de Google.

Es importante tener en cuenta que originalmente el propósito del formulario de radar de innovación fue medir el grado de innovación alcanzado por las micro y pequeñas empresas. Después de una investigación preliminar con startups que participaron en esta investigación, se descubrió que la mayoría de ellas encajan en este grupo (micro y pequeña empresa) y esta fue una de las razones para adoptar esta herramienta para la recopilación de datos.

Se explica que el formulario radar de innovación consta de 13 dimensiones: oferta, plataforma, marca, clientes, soluciones, relación, valor agregado, procesos, organización, cadena de suministro, presencia, red y ambiente. Cada dimensión se compone de un conjunto de variables y para cada variable se presentan tres alternativas con puntajes que van desde 1, 3 y 5 . La alternativa de peso 5 indica práctica sistémica, la alternativa de peso 3 indica práctica ocasional y alternativa de peso 1 indica poca o ninguna práctica para la variable considerada.

Además de definir el grado de innovación en las startups encuestadas, este estudio también obtuvo el grado en que las startups de tecnología de la información adoptaron tipologías y estrategias de innovación. Para medir este grado, se utilizó la misma metodología, de modo que las startups pudieran clasificarse como innovadores sistémicos, ocasionales o poco innovadores en las tipologías de innovación, así como practicantes sistémicos, practicantes ocasionales o practicantes pobres de estrategias de innovación.

Así, el grado de innovación en tipologías, estrategias y el grado general se obtuvo mediante la suma de los promedios encontrados en cada dimensión dividido entre el total de dimensiones, o sea 13 en total. De esta forma, las startups que presentaron un grado de innovación entre 1 y 2,9 puntos se clasificaron como poco o nada innovadoras, las que lograron un grado de innovación entre 3 y 3,9 puntos se clasificaron como innovadoras ocasionales y las que lograron 4 o más puntos fueron clasificados como innovadoras sistémicos de acuerdo con la metodología de aplicación radar de innovación (Sawhney, Wolcott, \& Arroniz, 2006; Bachmann, \& Destafani, 2008).

La tabla 1 presenta la información, basada en el radar de innovación, y utilizada para clasificar las prácticas innovadoras en las startups consultadas.

Tabla 1.

Grado de innovación

\begin{tabular}{|l|l|l|}
\hline \multirow{2}{*}{ Grado } & Clasificación \\
\cline { 2 - 3 } & Tipologías y estrategias. & Grado de innovación \\
\hline$\geq 4$ & Practicante sistémico & Innovador sistémico \\
\hline$\geq 3$ y $<4$ & Practicante ocasional & Innovador Ocasional \\
\hline$<3$ & Pequeño practicante & Poco innovador \\
\hline
\end{tabular}

Fuente: Elaboración propia (2019).

Es importante explicar que cuando se clasifica como un profesional sistémico o innovador en una estrategia de tipología y/o innovación particular, la startup a menudo aplica actividades y procedimientos relacionados con la tipología y/o estrategia considerada. Cuando se incluye en el grupo ocasional de 
profesionales y/o innovadores, la startupimplementa prácticas relacionadas con la tipología / estrategia en cuestión, de forma esporádica e inconstante. Finalmente, cuando se incluye en el grupo bajo o no practicante y/o innovador, la startup no implementa, o rara vez implementa, las actividades asociadas con una tipología y/o estrategia particular.

Como el objetivo principal de este estudio es presentar el grado de innovación en tipologías y estrategias de innovación utilizadas por startups de tecnología de la información (T.I) en el noreste de Brasil, se consideró como universo de investigación startups de TI ubicadas en el noreste y que figuran en la base de datos StartSe, 119 en total, tal como se destaca en la Tabla 2.

Tabla 2.

Muestra de estudio

\begin{tabular}{|l|c|c|}
\hline \multicolumn{1}{|c|}{ Estado } & $\mathbf{n}^{\circ}{ }^{\circ}$ startups registradas & $\mathbf{n}^{\circ}{ }^{\text {de startups participantes }}$ \\
\hline Alagoas & 3 & - \\
\hline Bahia & 24 & 6 \\
\hline Ceará & 21 & 7 \\
\hline Maranhão & 3 & 2 \\
\hline Paraiba & 7 & 3 \\
\hline Pernambuco & 20 & 4 \\
\hline Piauí & 2 & - \\
\hline Río Grande del Norte & 28 & 7 \\
\hline Sergipe & 11 & 5 \\
\hline Total & 119 & 34 \\
\hline
\end{tabular}

Fuente: Base de datos StartSe (2017)

El análisis de los datos se realizó utilizando estadísticas descriptivas, resultados porcentuales, variabilidad de los datos obtenidos (desviación estándar - DE), calificación y puntajes para tipologías y estrategias de innovación.

\section{PRESENTACIÓN Y ANÁLISIS DE DATOS}

\subsection{Caracterización de las Startups}

La Tabla 3 muestra los aspectos generales de las startups encuestadas. Como puede ver, la mayoría de las empresas tienen entre uno y siete años de operación. Este resultado corrobora parcialmente los postulados teóricos que indican que startups son empresas recién lanzadas y/o con un tiempo de trabajo corto (Dávila y Foster, 2005; Ciccione, 2014).

Tabla 3.

Aspectos generales de las startups participantes

\begin{tabular}{|l|l|c|c|}
\hline \multicolumn{1}{|c|}{ Aspectos generales } & \multicolumn{1}{|c|}{ Elementos analizados } & n. $^{\circ}$ de empresas & $\%$ \\
\hline \multirow{4}{*}{ Tiempo de funcionamiento } & Hasta 1 año & 3 & 8.8 \\
\cline { 2 - 4 } & Entre 1 y 3 años & 11 & 32,4 \\
\cline { 2 - 4 } & Entre 4 y 7 años & 14 & 41,2 \\
\cline { 2 - 4 } & Más de 7 años & 6 & 17,6 \\
\cline { 2 - 4 } & Total & 34 & 100 \\
\hline Mercado servido & Mercado local & 9 & 29,4 \\
\cline { 2 - 4 } & Mercado regional & 12 & 35,5 \\
\cline { 2 - 4 } & Nacional & 3 & 8.8 \\
\cline { 2 - 4 } & Internacional & 34 & 100 \\
\cline { 2 - 4 } & Total & 30 & 88,2 \\
\hline Facturación & Hasta 360,000 & 4 & 11,8 \\
\cline { 2 - 4 } & 360,000 a 3.6 millones & 34 & 100 \\
\cline { 2 - 4 } & Total & & 35 \\
\hline
\end{tabular}

Fuente: Elaboración propia (2019). 
En cuanto al mercado atendido por las startups que participan en este estudio, se notó un cierto equilibrio entre los mercados locales, nacionales y regionales. El mercado internacional fue el menos evidente, como se muestra en la Tabla 4. Se explica que el mercado local solo está cubierto por el estado de origen y operación de la startup, el mercado regional comprende las regiones geográficas de Brasil, mientras que el mercado internacional cubre las ubicaciones internacionales.

Con respecto a los ingresos, se observó que la mayoría de startups tienen ingresos brutos anuales de hasta $\mathrm{R} \$ 360,000.00$ (reales), lo que los caracteriza como micro y pequeñas empresas, de acuerdo con los criterios de la Ley Complementaria 123/2006 (Brasil, 2006).

\subsection{Principales tipos de innovación desarrolladas em startups}

En cuanto a los tipos de innovación, los resultados se presentan secuencialmente para las tipologías de producto, proceso, marketing e innovación organizacional, respectivamente. Como se muestra en la Tabla 4 , con respecto a la innovación de los productos, la mayoría de las startups investigadas se clasificaron en los grupos poco y ocasionalmente innovadores, 16 y 17 startups en cada grupo, respectivamente. Solo una startup se clasificó como innovadora sistémica en productos / servicios, esta empresa se encuentra en el estado de Rio Grande do Norte y su principal servicio es ofrecer contenido educativo en plataformas web y móviles.

Tabla 4.

Innovación de productos: distribución por estado

\begin{tabular}{|l|l|l|l|l|l|l|l|l|}
\hline \multirow{2}{*}{ Estado } & \multicolumn{3}{|c|}{$\begin{array}{c}\text { Innovador } \\
\text { sistémico }\end{array}$} & \multicolumn{2}{c|}{$\begin{array}{c}\text { Innovador } \\
\text { casual }\end{array}$} & \multicolumn{2}{c|}{$\begin{array}{c}\text { Poco } \\
\text { innovador }\end{array}$} & \multicolumn{2}{c|}{ Total } \\
\cline { 2 - 10 } & $\mathrm{n}^{\circ}$ & $\%$ & $\mathrm{n}^{\circ}$ & $\%$ & $\mathrm{n} .^{\circ}$ & $\%$ & $\mathrm{n}^{\circ}$ & $\%$ \\
\hline Bahia & 0 & 0 & 0 & 0 & 6 & 35,29 & 6 & 17,65 \\
\hline Ceará & 0 & 0 & 4 & 25 & 3 & 17,65 & 7 & 20,59 \\
\hline Maranhão & 0 & 0 & 1 & 6.25 & 1 & 5.88 & 2 & 5.88 \\
\hline Paraíba & 0 & 0 & 1 & 6.25 & 2 & 11,77 & 3 & 8.82 \\
\hline Pernambuco & 0 & 0 & 2 & 12,50 & 2 & 11,77 & 4 & 11,76 \\
\hline Rio Grande do Norte & 1 & 100 & 5 & 31.25 & 1 & 5.88 & 7 & 20,59 \\
\hline Sergipe & 0 & 0 & 3 & 18,75 & 2 & 11,76 & 5 & 14,71 \\
\hline Total & 1 & 100 & 16 & 100 & 17 & 100 & 34 & 100 \\
\hline
\end{tabular}

Fuente: Elaboración propia (2019).

Con respecto a la innovación de procesos, es importante tener en cuenta que ninguna de las startups encuestadas se clasificó como poco o no inventiva, como se muestra en la Tabla 5 . Se muestra de forma evidente que 23 empresas adoptan sistemáticamente prácticas relacionadas con la innovación de procesos, mientras que solo 11 ocasionalmente adoptan prácticas similares. En general, este resultado nos permite inferir quelas startups que participaron en este estudio han implementado innovaciones incrementales en sus productos a través de ajustes y mejoras en los procesos de creación / fabricación de sus productos / servicios.

Tabla 5.

Innovación de procesos: distribución por estado

\begin{tabular}{|l|l|l|l|l|l|l|}
\hline \multirow{2}{*}{ Estado } & \multicolumn{2}{c|}{ Innovador sistémico } & \multicolumn{2}{c|}{ Ocasional Innovador } & \multicolumn{2}{c|}{ Total } \\
\cline { 2 - 7 } & $\mathrm{n} .^{\circ}$ & $\%$ & $\mathrm{n} . .^{\circ}$ & $\%$ & $\mathrm{n}^{\circ}$ & $\%$ \\
\hline Bahia & 2 & 8.7 & 4 & 36,37 & 6 & 17,65 \\
\hline Ceará & 5 & 21,74 & 2 & 18,18 & 7 & 20,59 \\
\hline Maranhão & 2 & 8.7 & 0 & 0 & 2 & 5.88 \\
\hline Paraíba & 3 & 13.04 & 0 & 0 & 3 & 8.82 \\
\hline Pernambuco & 2 & 8.7 & 2 & 18,18 & 4 & 11,76 \\
\hline Rio Grande del Norte & 5 & 21,74 & 2 & 18,18 & 7 & 20,59 \\
\hline Sergipe & 4 & 17.38 & 1 & 9.09 & 5 & 14,71 \\
\hline Total & 23 & 100 & 11 & 100 & 34 & 100 \\
\hline
\end{tabular}

Fuente: Elaboración propia (2019). 
Se destaca las empresas de los estados de Rio Grande do Norte, Sergipe y Paraíba que alcanzaron el puntaje máximo de 5 puntos en innovación de procesos. Estas empresas ofrecen servicios a través de una plataforma web y móvil con contenido educativo personalizado, servicios de ingeniería y tecnología de la información, y servicios de plazas WiFi en espacios públicos, respectivamente.

De acuerdo con los datos obtenidos a través del formulario de recopilación de datos, las startups clasificadas como innovadoras de procesos sistémicos a menudo han adoptado nuevas tecnologías, e hecho uso de nuevos componentes, basados en las necesidades del cliente, así como ejecución, control y procesos modificados, y marketing para lograr una mayor eficiencia, calidad y flexibilidad, como recomiendan Ruzzier, Hojnik y Lipnik (2013).

Los resultados con respecto al tipo de innovación de marketing se pueden observar en la Tabla 6.

Tabla 6.

Innovación de marketing: distribución por estado

\begin{tabular}{|l|l|l|l|l|l|l|l|l|}
\hline \multirow{2}{*}{ Estado } & \multicolumn{2}{c}{$\begin{array}{c}\text { Innovador } \\
\text { sistémico }\end{array}$} & \multicolumn{2}{c|}{$\begin{array}{c}\text { Ocasional } \\
\text { Innovador }\end{array}$} & \multicolumn{2}{c|}{$\begin{array}{c}\text { Poco } \\
\text { innovador }\end{array}$} & \multicolumn{2}{c|}{ Total } \\
\cline { 2 - 10 } & $\mathrm{n} .^{\circ}$ & $\%$ & $\mathrm{n} .^{\circ}$ & $\%$ & $\mathrm{n}^{\circ}$ & $\%$ & $\mathrm{n}^{\circ}$ & $\%$ \\
\hline Bahia & 0 & 0 & 5 & 31.25 & 1 & 16,67 & 6 & 17,65 \\
\hline Ceará & 2 & 16,68 & 4 & 25 & 1 & 16,67 & 7 & 20,59 \\
\hline Maranhão & 1 & 8.33 & 1 & 6.25 & 0 & 0 & 2 & 5.88 \\
\hline Paraíba & 1 & 8.33 & 1 & 6.25 & 1 & 16,67 & 3 & 8.82 \\
\hline Pernambuco & 1 & 8.33 & 1 & 6.25 & 2 & 33,33 & 4 & 11,76 \\
\hline Rio Grande del Norte & 3 & 25 & 3 & 18,75 & 1 & 16,67 & 7 & 20,59 \\
\hline Sergipe & 4 & 33,33 & 1 & 6.25 & 0 & 0 & 5 & 14,71 \\
\hline Total & 12 & 100 & 16 & 100 & 6 & 100 & 34 & 100 \\
\hline
\end{tabular}

Fuente: Elaboración propia (2019).

Los datos obtenidos revelan que em la mayoría de startupsque participaron en esta investigación, 16 de las 34 consultadas, implementan ocasionalmente técnicas de innovación de marketing. Cabe destacar que, entre las 12 startups clasificadas como innovadoras sistémicas en marketing, ninguna alcanzó la puntuación máxima de 5 puntos, con la mejor posición alcanzando 4,5 puntos cada una. La startup ubicada en Maranhão es una startup de marketing y comunicación digital, mientras que la empresa ubicada en Rio Grande do Norte es una startup que ofrece contenido educativo en plataformas digitales.

En esta tipología de innovación, notamos un equilibrio entre los grados obtenidos por las startups participantes, especialmente en el grupo de startups poco innovadoras, en el que todas las startups lograron el mismo puntaje de 2.5 puntos de innovación de marketing.

Incluso haciendo referencia a las startups en el grupo de innovadores sistémicos en marketing, 12 en total, es importante tener en cuenta que la mayoría de ellas se concentran en los estados de Sergipe y Rio Grande do Norte, cuatro en Sergipe y tres en Rio Grande do Norte, tal como se presenta en la Tabla 7.

Finalmente, con respecto a la innovación organizacional, los datos revelan la constancia entre los grupos de startups que comprenden los innovadores sistémicos, los innovadores ocasionales y los grupos de pequeños innovadores, clasificandose 11, 12 y 11 startups en cada grupo, respectivamente. Este resultado también se puede ver en la Tabla 8 que muestra la distribución por estado de las startups en cada grupo.

Tabla 7.

Innovación organizacional: distribución por estado

\begin{tabular}{|c|c|c|c|c|c|c|c|c|}
\hline \multirow[t]{2}{*}{ Estado } & \multicolumn{2}{|c|}{$\begin{array}{l}\text { Innovador } \\
\text { sistémico }\end{array}$} & \multicolumn{2}{|c|}{$\begin{array}{l}\text { Innovador } \\
\text { casual }\end{array}$} & \multicolumn{2}{|c|}{$\begin{array}{l}\text { Poco } \\
\text { innovador }\end{array}$} & \multicolumn{2}{|c|}{ Total } \\
\hline & $\mathrm{n} .^{\circ}$ & $\%$ & $\mathrm{n}^{\circ}$ & $\%$ & $\mathrm{n}^{\circ}$ & $\%$ & $\mathrm{n} .^{\circ}$ & $\%$ \\
\hline Bahia & 2 & 20 & 2 & 15,39 & 2 & 18,18 & 6 & 17,65 \\
\hline Ceará & 3 & 30 & 2 & 15,38 & 2 & 18,18 & 7 & 20,59 \\
\hline Maranhão & 1 & 10 & 1 & 7.69 & 0 & 0 & 2 & 5.88 \\
\hline Paraíba & 1 & 10 & 1 & 7.69 & 1 & 9.09 & 3 & 8.82 \\
\hline
\end{tabular}


Continúa...

\begin{tabular}{|c|c|c|c|c|c|c|c|c|}
\hline \multirow[t]{2}{*}{ Estado } & \multicolumn{2}{|c|}{$\begin{array}{l}\text { Innovador } \\
\text { sistémico }\end{array}$} & \multicolumn{2}{|c|}{$\begin{array}{l}\text { Innovador } \\
\text { casual }\end{array}$} & \multicolumn{2}{|c|}{$\begin{array}{l}\text { Poco } \\
\text { innovador }\end{array}$} & \multicolumn{2}{|c|}{ Total } \\
\hline & $n .^{\circ}$ & $\%$ & $\mathrm{n} .^{\circ}$ & $\%$ & $\mathrm{n}^{\circ}$ & $\%$ & $n .^{\circ}$ & $\%$ \\
\hline Pernambuco & 1 & 10 & 1 & 7.69 & 2 & 18,18 & 4 & 11,76 \\
\hline $\begin{array}{l}\text { Rio Grande del } \\
\text { Norte }\end{array}$ & 1 & 10 & 3 & 23,08 & 3 & 27,28 & 7 & 20,59 \\
\hline Sergipe & 1 & 10 & 3 & 23,08 & 1 & 9.09 & 5 & 14,71 \\
\hline Total & 10 & 100 & 13 & 100 & 11 & 100 & 34 & 100 \\
\hline
\end{tabular}

Fuente: Elaboración propia (2019).

Cabe señalar que solo una startuplogró el puntaje máximo de 5 puntos en innovación organizacional, esta empresa se encuentra en el estado de Sergipe y ofrece servicios en ingeniería y tecnología de la información. A partir de los datos proporcionados, esta startup ha querido adoptar nuevas prácticas de gestión, incluida la gestión de residuos, la reorganización de actividades y la implementación de cambios radicales en la estrategia competitiva.

Otro punto que debe destacarse es que entre las 11 startups del grupo de innovadores sistémicos en aspectos organizativos, casi la mitad de ellas se concentran en los estados de Ceará y Bahía, 3 y 2 startups, respectivamente.

\subsection{Prácticas estratégica de innovación en startups}

La primera estrategia investigada fue la de asociación con clientes. La Tabla 8 presenta los resultados encontrados en las startups encuestadas.

Tabla 8.

Asociaciones de clientes: distribución por estado

\begin{tabular}{|l|l|l|l|l|l|l|l|l|l|}
\hline \multirow{2}{*}{ Estado } & \multicolumn{2}{|l|}{ Práctica sistémica } & \multicolumn{2}{l|}{ Práctica ocasional } & \multicolumn{2}{l|}{ Poca práctica } & \multicolumn{2}{l|}{ Total $^{\circ}$} \\
\cline { 2 - 10 } & $\mathrm{n} .^{\circ}$ & $\%$ & $\mathrm{n} .^{\circ}$ & $\%$ & $\mathrm{n}^{\circ}$ & $\%$ & $\mathrm{n}^{\circ}$ & ${ }^{\circ}$ \\
\hline Bahia & 2 & 8.33 & 3 & 33,34 & 1 & 100 & 6 & 17,6 \\
\hline Ceará & 5 & 20,83 & 2 & 22,22 & 0 & 0 & 7 & 20,5 \\
\hline Maranhão & 1 & 4.17 & 1 & 11.11 & 0 & 0 & 2 & 5.8 \\
\hline Paraíba & 3 & 12,5 & 0 & 0 & 0 & 0 & 3 & 8.8 \\
\hline Pernambuco & 3 & 12,5 & 1 & 11.11 & 0 & 0 & 4 & 11,7 \\
\hline Río Grande del Norte & 6 & 25 & 1 & 11.11 & 0 & 0 & 7 & 20,5 \\
\hline Sergipe & 4 & 16,67 & 1 & 11.11 & 0 & 0 & 5 & 14,7 \\
\hline Total & 24 & 100 & 9 & 100 & 1 & 0 & 34 & 100 \\
\hline
\end{tabular}

Fuente: Elaboración propia (2019).

La gran mayoría de las startups que participaron en esta encuesta fueron calificadas como profesionales sistémicos de asociaciones con clientes, de las 24 startups que indicaron que tenían actividades relacionadas con los clientes, 11 de ellas lograron una puntuación máxima de 5 puntos. A partir de los datos obtenidos, estas compañías han estado buscando información de manera intensiva sobre las necesidades, además de hacer un uso extensivo de los comentarios de los clientes para apoyar el desarrollo y lanzamiento de nuevos productos / servicios a través de asociaciones formales o informales, $y$ han implementado instalaciones y recursos, incluido el uso de herramientas de tecnología de la información, para mejorar las relaciones con sus consumidores.

También vale la pena mencionar que solo una startup se clasificó con poca práctica en actividades de asociación con clientes, esta es una startup con domicilio en Bahia que fabrica un modelo de mochila eléctrica con comando digital. Los resultados presentados pueden ser validados por Brunswicker y Vanhaverbeke (2015) quienes aclaran que sostienen que las asociaciones entre clientes y proveedores se producen a partir de actividades simples como la comunicación y la interacción entre la empresa y sus consumidores.

Otra estrategia verificada en las startups consultadas fue la de las asociaciones organizacionales. Los resultados obtenidos se presentan en la Tabla 9. 
Tabla 9.

Asociaciones organizacionales: distribución por estado

\begin{tabular}{|l|l|l|l|l|l|l|}
\hline \multirow{2}{*}{ Estado } & \multicolumn{2}{l|}{ Práctica sistémica } & \multicolumn{2}{l|}{ Práctica ocasional } & \multicolumn{2}{l|}{ Total } \\
\cline { 2 - 8 } & $\mathrm{n} .^{\circ}$ & $\%$ & $\mathrm{n} .^{\circ}$ & $\%$ & $\mathrm{n}^{\circ}$ & ${ }^{\circ}$ \\
\hline Bahia & 3 & 13,64 & 3 & 25 & 6 & 17,65 \\
\hline Ceará & 6 & 27,27 & 1 & 8.32 & 7 & 20,59 \\
\hline Maranhão & 2 & 9.09 & 0 & 0 & 2 & 5.88 \\
\hline Paraíba & 1 & 4.55 & 2 & 16,67 & 3 & 8.82 \\
\hline Pernambuco & 2 & 9.09 & 2 & 16,67 & 4 & 11,76 \\
\hline Rio Grande del Norte & 5 & 22,72 & 2 & 16,67 & 7 & 20,59 \\
\hline Sergipe & 3 & 13,64 & 2 & 16,67 & 5 & 14,71 \\
\hline Total & 22 & 100 & 12 & 100 & 34 & 100 \\
\hline
\end{tabular}

Fuente: Elaboración propia (2019).

Se notó que todas startups investigadas de manera sistemática u ocasional ya se han asociado con otras empresas y / o negocios relacionados. En total, se incluyeron 23 startups en el grupo de empresas que implementan sistemáticamente asociaciones con otras empresas para crear y entregar nuevos y mejores productos / servicios a los clientes, así como para ganar competitividad. Este hallazgo corrobora el pensamiento de Ghobril, Marcondes y Benedetti (2017), quienes informan que la asociación entre startups se lleva a cabo a favor del desarrollo de innovaciones y el aumento de la competitividad de las empresas participantes.

Destacamos que casi la mitad de las 23 startups que implementaron sistemáticamente asociaciones con otras empresas, 11 específicamente, se encuentran en los estados de Ceará y Rio Grande do Norte, con 6 y 5 startups en cada estado, respectivamente, como se muestra en la Tabla 10. Estos resultados se pueden asociar con el punto destacado tecnológico, especialmente con respecto a la presencia de startups en estos estados, ya que, en Ceará, por ejemplo, muchas startups reciben apoyo de bancos de financiación pública, institutos de investigación en salud, así como aceleradores y amplia red de coworkings locales (Segala, 2019). Lo más destacado dado a Rio Grande do Norte puede explicarse por la iniciativa de la Universidad Federal de Rio Grande do Norte (UFRN), a través del Instituto Digital Metropolis (IMD), que ha ofrecido instalaciones físicas y capacitación a empresas de base tecnológica (Jacomé, 2019).

Con respecto a la práctica de la innovación abierta, los resultados obtenidos en las startups encuestadas se presentan en la Tabla 10. Se explica oportunamente que la mayoría de las startups que participan en esta investigación, 15 de 34, se clasificaron en el grupo de poca práctica de la innovación abierta, con puntajes que van desde 2.2 a 2.83 puntos. En este grupo hubo un predominio de las startups de los estados de Bahía y Ceará, con 4 y 3 empresas en cada estado, respectivamente. Con respecto a los practicantes sistémicos de esta estrategia, hubo una concentración en el estado de Rio Grande do Norte.

Con respecto a la práctica de la innovación abierta, los resultados obtenidos en esta investigación confirman los hallazgos de Silva et al. (2019) de que, en las startups, existe una mayor disposición a aplicar elementos de asociaciones con clientes y otras organizaciones, y una menor predisposición a aplicación de la propiedad intelectual, amplia colaboración para la innovación y financiación pública.

Tabla 10.

Innovación abierta: distribución por estado

\begin{tabular}{|l|l|l|l|l|l|l|l|l|l|}
\hline \multirow{2}{*}{ Estado } & \multicolumn{2}{|l|}{ Práctica sistémica } & \multicolumn{2}{l|}{ Práctica ocasional } & \multicolumn{2}{l|}{ Poca práctica } & \multicolumn{2}{|c|}{ Total $^{\circ}$} \\
\cline { 2 - 10 } & $\mathrm{n} .^{\circ}$ & $\%$ & $\mathrm{n} .^{\circ}$ & $\%$ & $\mathrm{n} .^{\circ}$ & $\%$ & $\mathrm{n}^{\circ}$ & $\%$ \\
\hline Bahia & 1 & 14,29 & 1 & 8.33 & 4 & 26,67 & 6 & 17,65 \\
\hline Ceará & 0 & 0 & 3 & 25 & 4 & 26,67 & 7 & 20,59 \\
\hline Maranhão & 1 & 14,29 & 1 & 8.33 & 0 & 0 & 2 & 5.88 \\
\hline Paraíba & 1 & 14,29 & 2 & 16,67 & 0 & 0 & 3 & 8.82 \\
\hline Pernambuco & 0 & 0 & 1 & 8.33 & 3 & 20 & 4 & 11,76 \\
\hline Rio Grande del Norte & 3 & 42,84 & 2 & 16,67 & 2 & 13,33 & 7 & 20,59 \\
\hline
\end{tabular}


Continúa...

\begin{tabular}{|c|c|c|c|c|c|c|c|c|}
\hline \multirow[t]{2}{*}{ Estado } & \multicolumn{2}{|c|}{ Práctica sistémica } & \multicolumn{2}{|c|}{ Práctica ocasional } & \multicolumn{2}{|c|}{ Poca práctica } & \multicolumn{2}{|c|}{ Total } \\
\hline & n. ${ }^{\circ}$ & $\%$ & $\mathrm{n} .^{\circ}$ & $\%$ & n. ${ }^{\circ}$ & $\%$ & $\mathrm{n} .^{\circ}$ & $\%$ \\
\hline Sergipe & 1 & 14,29 & 2 & 16,67 & 2 & 13,33 & 5 & 14,71 \\
\hline Total & 7 & 100 & 12 & 100 & 15 & 100 & 34 & 100 \\
\hline
\end{tabular}

Fuente: Elaboración propia (2019).

Según los datos expuestos, 12 startups se clasificaron como practicantes ocasionales de innovación abierta, lo que demuestra que, aunque no es una práctica común, estas empresas finalmente han colaborado, asimilado y compartido conocimientos con el entorno externo.

Sin embargo, incluso con respecto a los datos en la Tabla 11, sólo 7 startups fueron clasificadas como practicantes sistémicos de innovación abierta, es decir, entre las 34 startups encuestadas sólo siete empresas a menudo han adoptado prácticas de colaboración, asimilado y compartido conocimientos y prácticas con entidades institucionales y económicas. También es oportuno destacar que, entre las siete empresas clasificadas como profesionales sistémicos de innovación abierta, dos de ellas alcanzaron la puntuación máxima de 5 puntos, estas empresas están domiciliadas en los estados de Bahía y Rio Grande do Norte. Además, entre las 7 startups con los puntajes más altos en innovación abierta, tres de ellas se encuentran en el estado de Rio Grande do Norte.

Finalmente, se examinó si la estrategia de internacionalización fue practicada por las startups de tecnología de la información residentes en el noreste de Brasil. Los resultados obtenidos se presentan en la Tabla 11.

Tabla 11.

Internacionalización: distribución por estado

\begin{tabular}{|l|l|l|l|l|l|l|l|l|}
\hline \multirow{2}{*}{ Estado } & \multicolumn{2}{l|l}{$\begin{array}{l}\text { Practica } \\
\text { sistémico }\end{array}$} & \multicolumn{2}{l|}{$\begin{array}{l}\text { Practica } \\
\text { casual }\end{array}$} & \multicolumn{2}{l|}{ Poca práctica } & \multicolumn{2}{l|}{ Total } \\
\cline { 2 - 11 } & n. ${ }^{\circ}$ & $\%$ & n. ${ }^{\circ}$ & $\%$ & n. ${ }^{\circ}$ & $\%$ & n. ${ }^{\circ}$ & $\%$ \\
\hline Bahia & 0 & 0 & 0 & 0 & 6 & 22,22 & 6 & 17,65 \\
\hline Ceará & 0 & 0 & 3 & 50 & 4 & 14,81 & 7 & 20,59 \\
\hline Maranhão & 0 & 0 & 0 & 0 & 2 & 7.41 & 2 & 5.88 \\
\hline Paraíba & 0 & 0 & 1 & 16,67 & 2 & 7.41 & 3 & 8.82 \\
\hline Pernambuco & 1 & 100 & 0 & 0 & 3 & 11.11 & 4 & 11,76 \\
\hline Rio Grande del Norte & 0 & 0 & 0 & 0 & 7 & 25,93 & 7 & 20,59 \\
\hline Sergipe & 0 & 0 & 2 & 33,33 & 3 & 11.11 & 5 & 14,71 \\
\hline Total & 1 & 100 & 6 & 100 & 27 & 100 & 34 & 100 \\
\hline
\end{tabular}

Fuente: Elaboración propia (2019).

En particular, la internacionalización fue la estrategia de innovación en la que el menor número de startups se clasificaron como practicantes sistémicos y ocasionales. Solo una startupubicada en el estado de Pernambuco que ofrece una plataforma digital para administrar eventos académicos y científicos ha indicado que con frecuencia exporta su servicio.

Yendo más allá, 6 startups fueron clasificadas como practicantes ocasionales de la estrategia de internacionalización, con énfasis para los estados de Ceará y Sergipe, con 3 y 2 startups cada una, respectivamente. Las compañías de Ceará trabajan con servicios relacionados con la plataforma industrial utilizando drones para inspecciones industriales, inteligencia artificial digital e Internet de las cosas, y plataforma en línea para contratar servicios de ingeniería y arquitectura. Los productos / servicios exportados por startups ubicadas en Sergipe incluyen la provisión de servicios en ingeniería y tecnología de la información y aplicaciones móviles.

Como se muestra en la Tabla 12, la mayoría de las startups encuestadas, 27 de 34, no realizan ninguna práctica asociada con la exportación de sus productos y servicios, centrándose en los mercados regionales y nacionales. Es necessária una explicación sobre esta estrategia de innovación, en la Tabla 3 fueron indicadas solamente 3 startupsque proven o mercado internacional, esta información difiere de los resultados exouestos aquí porque al responder las preguntas sobre el mercado de actuación, las startups marcaron el mercado 
principalmente atendido, em cuanto que en las preguntas que consideraron la práctica de la internacionalización, las startups pudieron señalar la exportacion, incluso en situaciones esporádicas.

Para comparar mejor las clasificaciones obtenidas para tipologías y estrategias de innovación, se preparó la Tabla 12 con el número de empresas agrupadas en cada grupo, de acuerdo con la leyenda: I. Prod. innovación de producto; I. Proc. - innovación de procesos; I. M - Innovación de marketing; I. O - innovación organizacional; PC - asociación con clientes; PO - asociaciones organizacionales; IA - Innovación abierta; Inter. Internacionalización.

Tabla 12

Resumen de resultados: tipologías y estrategias

\begin{tabular}{|l|l|l|l|l|l|l|l|l|}
\hline \multirow{2}{*}{ Practica } & \multicolumn{4}{l}{ Tipologías de innovación } & \multicolumn{3}{l|}{ Estrategias de innovación } \\
\cline { 2 - 10 } & I. Prod. & I. Proc. & I. M & IO & P.C & PO & IA & Inter. \\
\hline Sistémico & 1 & 23 & 12 & 11 & 24 & 22 & 7 & 1 \\
\hline De vez en cuando & 16 & 11 & 16 & 12 & 9 & 12 & 12 & 6 \\
\hline Poca práctica & 17 & 0 & 6 & 11 & 1 & 0 & 15 & 27 \\
\hline Total & 34 & 34 & 34 & 34 & 34 & 34 & 34 & 34 \\
\hline
\end{tabular}

Fuente: Elaboración propia (2019).

Específicamente a los resultados obtenidos de las estrategias de innovación, la Tabla 13 resume los principales beneficios asociados con cada una de estas estrategias, como se indica en más de una opción en el formulario de recolección de datos.

Tabla 13.

Beneficios de las estrategias de innovación.

\begin{tabular}{|l|l|l|l|l|l|l|l|l|}
\hline \multirow{2}{*}{ Beneficio } & \multicolumn{9}{|c|}{ Estrategia } \\
\cline { 2 - 9 } & $\begin{array}{l}\text { Asociación } \\
\text { con Clientes }\end{array}$ & $\begin{array}{l}\text { Asociación } \\
\text { Organizacional }\end{array}$ & $\begin{array}{l}\text { Innovación } \\
\text { abierta }\end{array}$ & \multicolumn{2}{l|}{ Internacionalizacion } \\
\cline { 2 - 9 } & $\mathrm{n} .^{\circ}$ & $\%$ & $\mathrm{n}^{\circ}$ & $\%$ & $\mathrm{n}^{\circ}{ }^{\circ}$ & $\%$ & $\mathrm{n}^{\circ}$ & $\%$ \\
\hline Aprovechar las ventas. & 22 & 64,7 & 27 & 79,4 & 23 & 67,6 & 5 & 14,7 \\
\hline $\begin{array}{l}\text { Adquirir conocimientos para } \\
\text { la optimización de procesos y } \\
\text { la creación de nuevos } \\
\text { productos/servicios. }\end{array}$ & 25 & 73,5 & 26 & 76,5 & 30 & 88,2 & 6 & 17,6 \\
\hline $\begin{array}{l}\text { Mejorar el rendimiento } \\
\text { empresarial y/o garantizar la } \\
\text { supervivencia empresarial. }\end{array}$ & 17 & 50,0 & 15 & 44,1 & 21 & 61,8 & 4 & 11,8 \\
\hline $\begin{array}{l}\text { Conocer nuevas técnicas de } \\
\text { gestión. }\end{array}$ & 2 & 5.9 & 13 & 38,2 & 1 & 2.9 & 2 & 5.9 \\
\hline $\begin{array}{l}\text { La empresa no reconoció los } \\
\text { beneficios o no aplicó esta } \\
\text { estrategia. }\end{array}$ & 1 & 2.9 & 0 & 0 & -- & ---- & 26 & 76,5 \\
\hline
\end{tabular}

Fuente: Elaboración propia (2019).

Como se muestra en la Tabla 13, el principal beneficio indicado por las startups que sistemáticamente o ocasionalmente adoptaron la estrategia de asociación con los clientes fue la adquisición de conocimiento para la optimización de procesos y la creación de nuevos productos / servicios, señalado por el $73.5 \%$ de las empresas, o sea 33 startups indicaron adoptar esta práctica estratégica.

Con respecto a la estrategia de las asociaciones organizacionales, el 79.4\% de startups encuestadas indicaron que percibían como beneficios, simultáneamente, el impulsar de las ventas y la adquisición de conocimiento para la optimización de procesos y la creación de nuevos productos / servicios. En cuanto a la innovación aberta, se destacaron dos beneficios: a) Adquisición de conocimiento para la optimización de procesos $\mathrm{y}$; b) impulsionamiento de ventas, con $73.5 \%$ y $88.2 \%$ respectivamente.

Finalmente, en la estrategia de internacionalización, la adquisición de conocimiento y el impulsionamiento de las ventas fueron indicados simultáneamente por el $17.6 \%$ de las startups que adoptan esta práctica de manera sistemática o ocasional. También vale la pena mencionar que conocer nuevas técnicas de gestión fue el beneficio menos indicado en todas las estrategias identificadas. Scotti (2002) corrobora este 
resultado y explica que las empresas adoptan nuevas técnicas de gestión destinadas a racionalizar y simplificar los procesos internos para mejorar la calidad como un paso preliminar en la práctica de exportación.

\subsection{Grado de innovación en startups de tecnología de la información en el Noreste de Brasil}

Presentado y explicado los puntajes de innovación en referencia a las tipologías y estrategias de innovación de cada una de startups que participan en este estudio, la Tabla 14 presenta el resultado general para el nivel de innovación de startups encuestadas, de acuerdo con la metodología del radar de innovación, así como sus clasificaciones respectivas por estado.

Tabla 14.

Clasificación de startups por estado y grupo de clasificación

\begin{tabular}{|c|c|c|c|c|c|c|c|c|}
\hline \multirow[t]{2}{*}{ Estado } & \multicolumn{2}{|c|}{ Innovadores sistémicos } & \multicolumn{2}{|c|}{$\begin{array}{l}\text { Innovador } \\
\text { ocasionales }\end{array}$} & \multicolumn{2}{|c|}{$\begin{array}{l}\text { Poco } \\
\text { innovador }\end{array}$} & \multicolumn{2}{|c|}{ Total } \\
\hline & $\mathrm{n} .^{\circ}$ & $\%$ & $\mathrm{n} .^{\circ}$ & $\%$ & n. ${ }^{\circ}$ & $\%$ & $\mathrm{n}^{\circ}$ & $\%$ \\
\hline Bahia & 0 & 0 & 4 & 19.05 & 2 & 28,57 & 6 & 17,65 \\
\hline Ceará & 1 & 16,67 & 6 & 28,57 & 0 & 0 & 7 & 20,59 \\
\hline Maranhão & 1 & 16,67 & 0 & 0 & 1 & 14,29 & 2 & 5.88 \\
\hline Paraiba & 0 & 0 & 2 & 9.52 & 1 & 14,29 & 3 & 8.82 \\
\hline Pernambuco & 0 & 0 & 3 & 14,29 & 1 & 14,29 & 4 & 11,76 \\
\hline Río Grande del Norte & 3 & 50 & 2 & 9.52 & 2 & 28,57 & 7 & 20,59 \\
\hline Sergipe & 1 & 16,66 & 4 & 19.05 & 0 & 0 & 5 & 14,71 \\
\hline Total & 6 & 100 & 21 & 100 & 7 & 100 & 34 & 100 \\
\hline
\end{tabular}

Fuente: Elaboración propia (2019).

Como generalmente se percibe, la mayoría de las startups que participaron en ese estudio, 21 empresas com mayor precisión, son innovadores ocasionales, lo que demuestra que aunque estas empresas reconocen e implementan innovaciones, estas se aplican de manera eventual y no sistemática.

\subsection{Media y Desviación estándar de los datos recopilados}

El grado medio y la desviación estándar en los datos recopilados se pueden ver en la Tabla 15.

Tabla 15.

Desviación media y estándar: tipologías y estrategias de innovación

\begin{tabular}{|l|l|c|c|}
\hline \multicolumn{2}{|l|}{ Tipologia } & Media & Desviacion estandar \\
\cline { 2 - 4 } & Innovación de producto & 2,82 & 0,66 \\
\cline { 2 - 4 } & Innovación de procesos & 3.98 & 0,58 \\
\cline { 2 - 4 } & Innovación de marketing & 3,42 & 0,59 \\
\cline { 2 - 4 } & Innovación organizacional & 3.19 & 0,72 \\
\hline \multirow{4}{*}{ Estrategias } & Asociación con organizaciones & 4.35 & 0,93 \\
\cline { 2 - 4 } & Asociación con clientes & 4.30 & 0,64 \\
\cline { 2 - 4 } & Innovación abierta & 3.21 & 0,84 \\
\cline { 2 - 4 } & Internacionalizacion & 1,47 & 0,97 \\
\hline Grado de innovación & 3,47 & 0.5 \\
\hline
\end{tabular}

Fuente: Elaboración propia (2019).

En general, cuando se evaluó como un grupo unitario, las startups encuestadas fueron calificadas como innovadoras medianas y ocasionales en innovación de procesos, innovación de marketing e innovación organizacional, mientras que para la tipología de innovación de productos, las startups fueron clasificados como no innovadoras. Con respecto a la variabilidad de los datos obtenidos, se observó que la mayor dispersión se asoció con la tipología de innovación organizacional, lo que indica que fue en esta tipología que 
los datos estaban más alejados del promedio identificado, por otro lado, fue en la tipología de innovación de procesos que los datos presentaron menos variabilidad.

Con respecto a las estrategias de innovación, se observó que, como grupo unitario, las startups se clasificaron como innovadores sistémicos para las estrategias de asociaciones organizacionales y ya asociaciones con clientes, obtuvieron un grado medio y clasificación como innovadores ocasionales para la estrategia de innovación abierta, y no fueron innovadores para la estrategia de internacionalización.

De este hallazgo queda claro una mayor variabilidad entre los datos y el promedio general obtenido, especialmente para las estrategias de asociación con otras organizaciones y la internacionalización. Además, se verificó que, en la estrategia de asociación con los clientes, se percibía la menor dispersión entre los datos recopilados, de acuerdo con la desviación estándar calculada.

Finalmente, em el análisis grupal, se notó que las startups encuestadas alcanzaron una calificación promedio de 3.47 puntos, por lo que se clasifican como practicantes innovadoras medianas y ocasionales, de acuerdo con la metodología del radar de innovación. Con respecto a la variabilidad, se observó que los datos recopilados, que dieron como resultado el grado de innovación de estas empresas, indicaron la variabilidad media entre los grados obtenidos.

\section{CONCLUSIONES Y RECOMENDACIONES}

Esta investigación tuvo como objetivo presentar el grado de innovación en tipologías y estrategias de innovación utilizadas por startups de tecnología de la información en el noreste de Brasil. Específicamente, identificar las principales tipologías de innovación, así como el grado alcanzado por startups de tecnología de la información.

En cuanto a las tipologías, los resultados mostraron que la innovación de procesos fue la tipología más evidente en las startups consultadas, ya que 23 de las 34 empresas encuestadas se incluyeron en el grupo de prácticas sistémicas para esta tipología. En general, las empresas sistémicas para esta práctica se concentraron en el estado de Rio Grande do Norte.

Los resultados indican que las asociaciones de clientes y las asociaciones organizacionales son las estrategias más adoptadas por las startups de tecnología de la información, de modo que 24 y 23 empresas se clasificaron como innovadoras sistémicas, respectivamente, en estas estrategias.

Específicamente, la estrategia de las asociaciones organizacionales mostro que las empresas que implementan sistemáticamente asociaciones con otras organizaciones buscan apoyo para la creación y el suministro de nuevos y mejores productos / servicios a los clientes, y tratan de lograr una mayor competitividad. Es pertinente enfatizar que entre las startupssistémicas en asociación con clientes y en asociación con otras organizaciones, la mayoría de ellas se encuentran en los estados de Ceará y Rio Grande do Norte.

Aún con referencia a las estrategias de innovación, se observó que sólo siete empresas han alcanzado el grado de la práctica sistémica para la estrategia de innovación aberta, siendo la mayoria classificadas como poco inovadoras en esta practica, revelando de esta forma que esos negócios deben buscar e implementar asociaciones com grupos mas grandes y diversos de agentes externos, especialmente agencias gubernamentales, instituciones de investigación, entidades cooperativas y entidades paraestatales.

Específicamente para el grado global de innovación, de acuerdo con la metodología del radar de innovación, los resultados obtenidos indicaron que la mayoría de las startups encuestadas, $61.76 \%$ más precisamente, fueron clasificadas en el grupo de innovadoras ocasionales, incluyendo el grado medio de innovación para estas startups, consideradas como un grupo unitario, fue de 3,47 puntos, enfatizando la característica mediana de implementación y gestión de la innovación en estas empresas.

De los resultados demostrados, se concluye que la mayoría de las empresas consultadas implementan estrategias de asociación con clientes y asociaciones con otras organizaciones. Las startups del noreste estan deacuerdo al indicar la busca de asociaciones para adquirir conocimientos y por lo tanto optimizar procesos y crear nuevos productos y servicios. Se diferencian por la intensidad en la que implementan estrategias de asociación con clientes, asociaciones con otras organizaciones, innovación abierta e internacionalización.

La principal limitación de este estudio fue el tamaño de la muestra. La muestra alcanzada no permitió el desarrollo de mayores inferencias estadísticas entre el grado y las estrategias de innovación adoptadas. Outra limitación puede ser indicada por la herramienta de medición para el grado de innovación, el formulario radar de innovación, que a pesar de ser um instrumento validado y ampliamente utilizado por el Servicio Brasileño de Apoyo a las Micro y Pequeñas Empresas (SEBRAE), e incluso ademas de las implementaciones realizadas, es posible que esta herramienta no haya cubierto todos los aspectosrelacionados con las startups, como la medición rápida del crecimiento, la implementación de 
modelos comerciales escalables y replicables, la diferenciación concreta de las innovaciones propuestas, así como un mayor enfoque en los aspectos técnicos relacionados com el uso de la tecnología de la información la startup como insumo básico para la operación de estas empresas.

Además, como no se encontraron estudios que analizaran el grado de innovación en las startups, no fue posible implementar mayores asociaciones entre los resultados y los postulados teóricos. Por esta razón, la principal contribución teórica de este estudio es presentar un retrato empírico de las principales tipologías, estrategias y grado de innovación alcanzado por startups de tecnología de la información.

En consecuencia de las limitaciones presentadas, se sugieren otros estudios con un número mayor de startups, con el fin de obtener mayores inferencias estadísticas. Otra sugerencia es el uso de una herramienta diferente para medir el grado de innovación, como el cuestionario de diagnóstico de innovación - QDI. El uso de otra herramienta de medición permitirá agregar comparaciones y hallazgos a los resultados indicados en esta investigación.

\section{REFERÊNCIAS}

ABSTARTUPS - Associação Brasileira de Startups. Consultoria Accenture. (2018). O Momento da startup brasileira e o futuro do ecossistema de inovação. Disponível em:

<https://abstartups.com.br/PDF/radiografia-startups-brasileiras.pdf>. Acesso em: 30. Out. 2018.

Agrawal, A., Bhattacharya, S. \& Hasija, S. (2016). Cost-Reducing Innovation and the Role of Patent Intermediaries in Increasing Market Efficiency. Production and Operations, Management Society, 25(2), 173-191, 2016.

Alberti, F. G, Pizzurno, E. (2017). Oops, I did it again! Knowledge leaks in open innovation networks with startups. European Journal of Innovation Management, 20(1), 50-79.

Andrade, A. P. V., Lins Filho, M. L., Silva, G. G. (2017). Capacidade de inovar em startups: Uma abordagem sob a ótica da orientação para a aprendizagem. In: XL Encontro da ANPAD, 2016, Costa do Sauipe. Anais eletrônicos... Costa do Sauípe, 2016. Recuperado de < http://www.anpad.org.br/ anpad/abrir_pdf.php?e=MjE1Mjk= > .

Bachmann, D. L., \& Destefani, J. H. (2008). Metodologia para estimar o grau das inovações nas MPE. Curitiba: [s.n.].

Belkahla, W., Triki, A. (2011). Customer knowledge enabled innovation capability: proposing a measurement scale. Journal of knowledge management, 15(4), 648-674.

Berne, D. F. (2016). O Grau de Inovação das Indústrias MPE da Região Metropolitana Oeste e Sudoeste de São Paulo. Dissertação (Mestrado em Administração). Faculdades Campo Limpo Paulista. São Paulo.

Blank, S., \& Dorf, B. (2012). The Startup Owner's Manual: The Step by Step Guide for Building a Great Company. California: K\&S Ranch.

BRASIL. Lei no 123/2006, de 14 de dezembro de 2006. (2006). Institui o Estatuto Nacional da Microempresa e da Empresa de Pequeno Porte. Diário Oficial da república Federativa do Brasil. Brasília, DF, 14 dez. 2006. Disponível em: <http://www.planalto.gov.br/ccivil_03/leis/LCP/Lcp123.htm>. Acesso em: 11 dez. 2017.

Brunswicker, S., Vanhaverbeke, W. (2015). Open innovation in small and medium-sized enterprises (SMEs): external knowledge sourcing strategies and internal organizational facilitators. Journal of Small Business Management, 53(4), 1241-1263.

Brunswicker, S.; Vanhaverbeke, W. (2015). Open innovation in small and medium-sized enterprises (SMEs): external knowledge sourcing strategies and internal organizational facilitators. Journal of Small Business Management, 53(4), pp. 1241-1263.

Chesbrough, H. (2012). Inovação aberta: como criar e lucrar com a tecnologia. Porto Alegre: Bookman, 241 p.

Cicconi, E. G. (2014). Proposta de um modelo de tomada de decisão de investimento por parte dos fundos de venture capital em empresas start-ups de biotecnologia no Brasil. 2014, 140 f. Tese (Doutorado em Administração) - Faculdade de Administração, economia e contabilidade, Universidade de São Paulo, São Paulo.

Cropley, D. H., Kaufman, J. C. \& Cropley, A. J. (2011). Measuring Creativity for Innovation Management. Journal of Technology Management \& Innovation, 6(3).

Crossan, M. M., \& Apaydin, M. (2010). A Multi-Dimensional Framework of Organizational Innovation: A Systematic Review of the Literature. Journal of Management Studies, 47(6), 1154-1191.

Cruz, M. R., Camargo, M. E. Braz, P. H. C., Frey, E. H., \& Frey, M. V. (2015). Estratégias para inovação na cadeia produtiva da maçã brasileira. Revista Raunp, 8 (1), p. 64-73, 2015.

Davila, T. \& Foster, G. (2005). Startup firms growth, management control systems adoption, and performance. In: AAA Management Accounting Section Meeting Paper. 
Edison, H., Bin Ali., N., \& Torkar, R. (2013). Towards innovation measurement in the software industry. Journal of Systems and Software, 86(5), 1390-1407.

Freire, C. T., Maruyama, F. M., \& Polli, M. (2017). Inovação e empreendedorismo: Políticas Públicas e Ações Privadas. Novos estudos, 36(3), 51-76.

Ganotakis, P. \& Love, J. H. (2011). R\&D, product innovation, and exporting: Evidence from UK new technology based firms. Oxford Economic Papers, 63, 279-306.

Ghobril, A. N., Marcondes, R. C \&, Benedetti, M. (2017). Inovação por meio da integração entre corporações, ICTs e startups. Fórum Liberdade Econômica, Centro de Liberdade Econômica, Mackenzie.

Glückler J. (2013). Knowledge, networks and space: Connectivity and the problem of non-interactive learning. Regional Studies, 47, 880-894.

Heidenreich, S. \& Kraemer, T. (2016). Innovations-Doomed to Fail? Investigating Strategies to Overcome Passive Innovation Resistance. Journal of Product Innovation Management, 33(3), 277-297.

Hsieh, C.-T., Lee, W.-L \& Huang, H.-C. (2016). Using transaction cost economics to explain open innovation in start-ups. Management Decision, 54(9), 2133-2156.

Jacomé, I. (2019). Após dois anos, projeto que quer transformar Natal em pólo tecnológico reúne 46 empresas e mais de 700 empregos. Disponible: <https://g1.globo.com/rn/rio-grande-donorte/noticia/2019/08/11/apos-dois-anos-projeto-que-quer-transformar-natal-em-polo-tecnologicoreune-46-empresas-e-mais-de-700-empregos.ghtml>. Acceso en: jan. 2020.

Jardón, C. M. (2012). Determinantes de la capacidad de innovación em PYMES regionales. Universidade de Vigo. Departamento de economia aplicada. Working paper: 12/02, Sept., 2012. Disponível em: <http://webs.uvigo.es/x06>. Acessado em: jan. 2018.

Keinz, P., Hienerth, C. \& Lettl, C. (2012). Designing the Organization for user-driven innovation. Journal of Organizational Design, 1(3), 20-36.

Marion, T. J., Friar, J. H., \& Simpson, T. W. (2012). New product development practices and early-stage firms: Two in-depth case studies. Journal of Product Innovation Management, 29(4), 639-654.

Mas-Tur, A., Pinazo, P., Tur-Porcar, A. M. \& Sánchez-Masferrer, M. (2015). What to avoid to succeed as an entrepreneu. Journal of Business Research, 68, 2279-2284.

MCTI, Ministério De Ciência, Tecnologia, Inovações e Comunicações. (2017).

Padrão, L. C., \& Andreassi, T. (2013). O desempenho de startups de base tecnológica: um estudo comparativo em regiões geográficas brasileiras. Revista da Micro e Pequena, 7 (2), 66-79.

Ren, S.; Eisingerich, A. B., \& TSAI, H-T. (2015). How do marketing, research and development capabilities, and degree of internationalization synergistically affect the innovation performance of small and mediumsized enterprises (SMEs)? A panel data study of Chinese SMEs. International Business Review, 24, 642651.

Ries, E. (2012). A Startup Enxuta: como os empreendedores atuais utilizam a inovação contínua para criar empresas extremamente bem-sucedidas. Lua de Papel, $1^{\text {a }}$ edição. São Paulo-SP.

Robehmed, N. (2013). What is a Startup? Forbes. Recuperado de: <www.forbes.com/sites/natalierobehmed/2013/12/16/what-is-astartup/\#5c77b0c64c63>.

Rocha, R. O., Olave, M. E. L., \& Ordonez, E. D. M. (2019). Estratégias de Inovação para Startups. Revista Pretexto, 20(2), 87-99.

Ruzzier, M., Hojnik, J. \& Lipnik, A. (2013). Relationship between innovation and internationalization of slovenian internationalized companies. In: Industry, Science and Police Markers for Sustainable Future, Slovenia. Recuperado de: <https://ideas.repec.org/h/mgt/micp13/579-595.html>.

Saebi, T.\& Foss, N. J. (2014). Business models for open innovation: matching heterogenous open innovation strategies with business model dimensions. Center for Service Innovation-Department of Strategy and Management Norwegian School of Economics, $45 \mathrm{f}$.

Sawhney, M., Wolcott, R. C. \& Arroniz, I. (2006). The 12 different ways for companies to innovate. MIT Sloan Management Review, 47(3), 75-81.

Scotti, M. A. (2002). Implementação de estratégias de internacionalização de empresas: um processo de aprendizagem organizacional. Dissertação (Mestrado em Administração) - Fundação Getulio Vargas, São Paulo, 2002.

Segala, M. (2019). Rapadura Valley? 5 polos de empreendedorismo que ganham força no Brasil. Conheça cidades que proporcionam as condições ideais para o surgimento de startups de sucesso. Disponible: $<$ https://revistapegn.globo.com/Startups/noticia/2019/01/rapadura-valley-5-polos-deempreendedorismo-que-ganham-forca-no-brasil.html>. Acceso en: jan. 2020.

Shaw, G. \& Williams, A. (2009). Knowledge transfer and management in tourism organisations: an emerging research agenda. Tourism Management, 30, 325-335. 
Shontell, A. (2014). This is the definitive definition of a startup, Business Insider UK. Recuperado de: $<$ http://uk.businessinsider.com/what-is-a-startup-definition-2014-12? r=US\&IR=T>.

Silva, A. D., Pereira, L. G., Sousa, R. R., Bispo, R. M. A., \& Silva, L. R. (2019). Inovação Aberta em Startups de Economia Compartilhada: uma análise em empresas brasileiras. Iniciação - Revista de Iniciação Científica, Tecnológica e Artística, 7(3), pp. 1-17.

STARTSE: O programa. (2017). Disponível em: <https://www.startupbrasil.org.br/sobre_programa/>. Acesso em: Mar. 2017.

Stoilov, I. A. (2015). Innovation in technological start-ups: Korean start-up ecosystem. Universitat Autonoma de Barcelona - Degree: Business Administration and Management. 